\title{
Treatment Experiences of Individuals Born with Cleft Lip and/or Palate in the UK
}

\author{
Aidan Searle*, Shabnum Ali, Scott Deacon and Andrea Waylen
}

School of Oral \& Dental Sciences, University of Bristol Dental School \& Hospital, UK

*Corresponding author: Aidan Searle, School of Oral \& Dental Sciences, University of Bristol Dental School \& Hospital, Lower Maudlin Street, Bristol BS1 2LY, UK, Tel: 0117 3421768, E-mail: A.J.Searle@bristol.ac.uk

\begin{abstract}
Despite receiving treatment by specialist services in childhood and adolescence some individuals report ongoing cleft-related issues that impact on their self-confidence and quality of life due to both aesthetic and functional reasons.

This study used in-depth qualitative interviews to explore 1) Experiences of specialist treatment during childhood and adolescence for individuals born with CLP and 2) ReEngagement with specialist cleft services as an adult in the U.K. Thematic analysis of the interview data led to the emergence of four key themes: 'Living with a cleft as an adult', 'Re-engagement with treatment', 'Expectations of treatment and communication', and 'Health professionals' awareness of cleft'. It was apparent that many individuals were dissatisfied with specialist treatment in childhood or had not felt involved in making decisions about treatment. It was also clear that non-specialist professionals may lack awareness of the issues individuals born with CLP face in relation to living with CLP and re-engagement with specialist cleft services in the U.K. The implications of the findings are discussed in relation to future research and training both specialist and non-specialist health professionals such as GMPs and GDPs.
\end{abstract}

\section{Keywords}

Cleft Lip and/or Palate (CLP), Qualitative, Cleft Treatment Pathway, General Dental Practitioner (GDP), General Medical Practitioner (GMP)

\section{Introduction}

Cleft Lip and Palate (CLP) is a common congenital condition with a global incidence of approximately $1 / 700$ live births [1-3]. In the United Kingdom around 1200 babies are born with a cleft annually [4,5]. Providing care for a child born with CLP can be complex and is likely to require a wide range of treatments from birth to Twenty-One years of age - the 'cleft treatment pathway'. The aim of treatment is to restore anatomy (face and dentition) and function (hearing, speech and feeding) and to encourage the physical and psychological development of the child born with CLP. The Clinical Standards Advisory Group reported that care for a child born with CLP was, at that time, provided by 76 surgeons in 57 centres in the United Kingdom (UK) [6]. Furthermore, treatment outcomes from some UK cleft centres were shown to be less satisfactory than those achieved elsewhere in Europe. Consequently, a series of recommendations were made with the aim of improving child health outcomes. These included centralisations of cleft services and care provision in the United Kingdom via the development of Multidisciplinary Teams (MDTs). It is also considered that the best and most cost-effective outcomes for patients and clients are achieved when professionals work together, learn together, engage in clinical audit of outcomes together, and generate innovation to ensure progress in practice and service. Specialist health professionals such as orthodontists, speech therapists, audiologists and psychologists work together with surgical colleagues in delivering cleft services. There are also putative benefits of service provision via MDTs within a centralised cleft service. For example, training under consultant supervision in a centralised system is likely to improve as expertise can be shared directly with clinical colleagues. Furthermore, critical incident reporting, guideline development, audit and research can inform the delivery of care [7]. Therefore, this model was advocated by the Clinical Standards Advisory Group report [3] in order to try and reduce the disparity in outcomes for children born with CLP in the United Kingdom compared to those born in Europe, particularly Scandinavia.

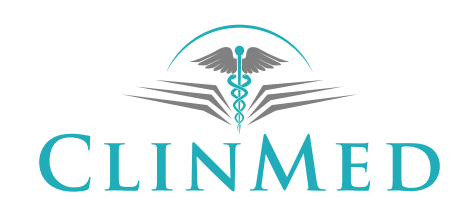

INTERNATIONAL LIBRARY
Citation: Searle A, Ali S, Deacon S, Waylen A (2017) Treatment Experiences of Individuals Born with Cleft Lip and/or Palate in the UK. Int J Oral Dent Health 3:042. doi.org/10.23937/2469-5734/1510042 Received: October 26, 2016: Accepted: May 13, 2017: Published: May 15, 2017

Copyright: (c) 2017 Searle A, et al. This is an open-access article distributed under the terms of the Creative Commons Attribution License, which permits unrestricted use, distribution, and reproduction in any medium, provided the original author and source are credited. 
However, despite access to this multidisciplinary approach to treatment individuals born with CLP may face ongoing issues in adulthood that impact on self-confidence, inter-personal relationships, and achievement in education and employment [8-11]. The ongoing impact of living with CLP may, in part, be a function of dissatisfaction with treatment or unmet aesthetic or functional needs. However, one recent qualitative study with 52 adults born with CLP found that some participants questioned the value and necessity of ongoing treatment. They suggested that decision-making was influenced by other peoples' opinions about their appearance and function and consequently led to the exacerbation of confusion and distress [8]. Furthermore, individuals born with CLP may also experience positive psychological growth and wellbeing. Psychological growth and wellbeing may enhance individuals' perceived autonomy and competence with regard to making decisions to engage or disengage with treatment of their own volition [12].

Nonetheless, it is also likely that some individuals born with CLP will be discharged from specialist services before twenty-one years of age. However, if individuals born with CLP disengage from treatment before being clinically discharged from the 'cleft treatment pathway', they may, at a later date, experience difficulties in accessing and/or re-engaging with specialist treatment services. For example, individuals born with CLP may experience continuing issues with breathing or nasal symptoms [13], and impaired speech and hearing $[14,15]$. It has been suggested that General Medical and Dental Practitioners (GMPs and GDPs) differentiate real and perceived needs and be able to refer to appropriate specialist services [16]. Furthermore, an audit conducted in 2004 by the West Midlands Cleft Service in the U.K. reported that 80 of 151 adult patients (55\%) born with CLP underwent surgery as part of their management plan and 36 (25\%) more were awaiting surgery. Of those referred for surgery only $22(19 \%)$ came from primary care: 20 from GMPs and two from GDPs. Fifty percent of adult patients referred to the West Midlands Cleft Service required restorative dental treatment and/ or referral to orthodontist services [17].

Health professionals such as GMPs and GDPs are likely to see individuals born with CLP on a regular basis as part of their practice. However, there may be a lack of understanding on their part with regard to oral health issues and opportunities for ongoing treatment for individuals born with a cleft. At present, there is little evidence (other than audit) of the impact of the treatment for individuals born with CLP that are no longer receiving specialist treatment, particularly those living in the United Kingdom [18]. Moreover, there are no studies that have included the personal experiences of re-engaging with cleft services in interviews with adults born with CLP.
Recent qualitative research has highlighted the psychosocial challenges faced by older adults born with CLP [16]. Individuals born with CLP felt that health services could be more considerate to older individuals born with CLP particularly around dentistry and information provision. However, this study was conducted with telephone interviews with adults aged $57-82$ years so findings may not represent experiences of younger and middle aged adults leading lives without the support of specialist cleft services. This paper also highlighted aesthetic and confidence issues more so that than functional issues associated with individuals born with CLP. It would appear that the post-treatment issues associated with adults living with CLP are a neglected area in qualitative CLP research and a paucity of data collected in face to face settings. There is also a need to raise awareness of both these issues amongst non-specialist health professionals if adults born with CLP are to successfully re-engage with specialist services.

Therefore, the aims of this qualitative study were twofold; to explore: 1) Experiences of specialist cleft treatment during childhood and adolescence for individuals born with CLP and 2) Re-Engagement with specialist cleft services as an adult in the U.K.

\section{Methods}

\section{Procedure}

The qualitative data was collected as part of the development an online resource for families and individuals affected by CLP - the www.healthtalk.org 'Cleft Lip and Palate' module. www.healthtalk.org provides 'free, reliable information about health issues, by sharing individuals' experiences of living with a wide variety of health conditions: http://www.healthtalk.org/about/overview).

This programme of research was guided by an advisory panel comprising lay people with experience of CLP, health professionals and academics assisted in the development of the module and interview topic guides. Due to the aims of informing the cleft lip and palate module for www.healthtalk.org, the topic guide for individuals born with CLP included the following topics: personal and family background, school experience, everyday life, cleft treatment, social experience, post school experience and employment, strategies for overcoming cleft related difficulties (Appendix 1). The present study draws on topics that have particular relevance to treatment experiences, re-engagement with treatment and public and professional awareness of issues pertaining to individuals born with CLP.

The guide for the present study included topics pertinent to adult life with CLP and disengagement from or re-engagement with cleft services in the U.K. In-depth interviews were conducted in two parts (Appendix 1). First, the participant was invited to tell their story of living with CLP. Second, the topic guide was used to fur- 
ther probe and prompt individuals to generate richer data about their experiences.

Individuals were recruited through multiple methods including recommendations from lay panel members, social media platforms and support organisations such as CLAPA (Cleft Lip and Palate Association), www.healthtalk.org, and Adult Voices (a council for adults born with CLP), and presentations at Special Interest Group meetings (SIG: Nurses, Psychologists). A total of 22 individuals born with CLP were approached for interview and a further 3 individuals were also parents of children born with CLP. All individuals were sent an information sheet via email by AS. The information sheet included details of ethical approval and the intended use of data. Ethical approval for the study was granted to www.healthtalk.org/Health Experiences Research Group, University of Oxford by Berkshire Research Ethics Committee for health research. The approval permits www.healthtalk. org/Health Experiences Research Group to recruit participants, video and audio record interviews, and present both textual and video data on the on www.healthtalk.org modules, and other publications.

Participants were given a period of 1-2 weeks before they were re-contacted by AS for their consent to be interviewed either by video or audio only. Of these, 15 individuals participated in an interview for this aspect of the study, and mot interviews were conducted within 4 weeks of receipt of information sheet. Seven participants did not participate in an interview due to difficulties in finding a time and location for the interview due to family and/or employment commitments, or did not respond to attempts to contact by email and telephone following receipt of study information.

Individuals gave written consent to take part and the consent form stated that they could withdraw from the interview or withdraw their data from the study follow- ing an interview. Interview transcripts were returned to individuals to allow them the opportunity to review their data. There were no withdrawals from interviews or withdrawal of data, however one participant deleted data pertaining to a particular event experienced in the care of the health service.

\section{Participants}

Interviews were conducted with 9 females (18-53 years) and 5 men (17-62 years) who were born with cleft and/or palate (Table 1). All individuals were White-British/Irish, although one individual received cleft treatment as a child in his native Canada.

The lead author (AS) travelled to meet individuals in their homes in various regions of the U.K. and 12 interviews were conducted 'face to face' and video-recorded. For pragmatic reasons and/or individual preference three interviews were conducted via telephone and audio-recorded. The interviews were between 22 minutes and 66 minutes long, the mean duration of interview was 55 minutes.

\section{Analysis}

All transcripts of the audio data from the interviews with 15 individuals born with CLP were transcribed verbatim. A thematic analysis was then undertaken following the guidelines for thematic analysis stipulated by Braun \& Clarke [19]. Thematic analysis is a flexible and useful research tool for psychological research, which can potentially provide a rich and detailed, yet complex account of data [19].

First, the analysts $A S$ and $S A$ read and reread the transcripts to familiarise themselves with the dataset. Second, initial codes were identified and a coding frame was developed on a sub-sample of 4 interviews by AS and SA. Discrepancies in codes were discussed by the analysts until consensus was reached a definitive cod-

Table 1: Individuals born with cleft lip and/or palate $(N=15)$.

\begin{tabular}{|l|l|l|l|l|}
\hline $\begin{array}{l}\text { Individual } \\
\text { Gender/Age }\end{array}$ & Cleft Type & Marital Status & Occupation & Ethnicity \\
\hline Male, 62 years & $\begin{array}{l}\text { Cleft lip, son and grandson also born with } \\
\text { Cleft }\end{array}$ & Single & Docker & White British \\
\hline Male, 23 years & $\begin{array}{l}\text { Cleft lip and palate, son born with cleft lip } \\
\text { and palate }\end{array}$ & Cohabiting & Lifeguard & White British \\
\hline Female, 25 years & Cleft lip and palate & Married & Graduate/Call centre worker & White British \\
\hline Female, 24 years & Cleft palate & Single & Teacher & White British \\
\hline Female, 22 years & Cleft lip and palate & Single & HE Student & White British \\
\hline Male, 41 years & Cleft lip and palate & Married & Lecturer & White Canadian \\
\hline Female, 47 years & Cleft lip and palate & Married & Self-employed/Street pastor & White British \\
\hline Male, 38 years & Cleft lip and palate & Single & Self-employed Arts promoter & White British \\
\hline Female, 19 years & Cleft lip and palate & Single & Nursing student & White British \\
\hline Female, 39 years & Cleft lip and palate & Single & Teacher & White Irish \\
\hline Male, 17 years & Cleft lip and palate & Single & FE Student & White -British \\
\hline Female, 19 Years & Cleft lip and palate & Single & HE Student & White British \\
\hline Female, 53 years & $\begin{array}{l}\text { Cleft lip and palate, daughter born with cleft } \\
\text { lip and palate }\end{array}$ & Single & Teacher & White British \\
\hline Female, 18 years & Born with cleft lip and palate & Single & Student/Childcare worker & White British \\
\hline Male, 20 years & Cleft lip and palate & Single & Retail manager & White British \\
\hline
\end{tabular}


ing frame was generated. All transcripts were then coded using NVivo, Version 9. Third, once all the transcripts had been coded AS and SA searched the coded transcripts for emerging themes. Fourth, the emerging themes were reviewed and discrepancies were discussed to ensure there was agreement and that no new themes were emerging from the data. Interpretation of the coded interview data was undertaken by AS, AW, and SA and themes emerging from the data were identified.

\section{Results}

Four key themes emerged from the interview data analysis:

1. Living with a cleft as an adult (including professional support pre- and post-treatment)

2. Re-engagement with treatment as an adult (for aesthetic and functional reasons)

3. Expectations of treatment and communication

4. Health Professionals' awareness of cleft (GMPs, GDPs and referral to specialist services)

\section{Living with a cleft as an adult}

Individuals' experiences of treatment during childhood and adolescence were explored to provide context to their experiences of treatment as an adult. While it is understandable that treatment in infancy and childhood is facilitated through parents and health professionals, treatment during adolescence also seemed to take place with minimal input from the young person him/herself.

As I got older you know, they [HCPs] started to ask me what I wanted done and, do you know what I mean, it kind of turned around. But, yeah, l'd say at high school it was still very much on them on their part, yeah. I don't really feel like I had a choice. Like I think in appointments they would suggest things and it almost wasn't given to me as an option, it was like, "This is what I think we need to do next". So, I just thought, "OK, well that's what I've got to do", so I think I had another palate repair. And then after that it was very much a cosmetic thing, so rhinoplasty, implants. I had my lip taken apart again and they re-sewed the lip line. (Female, 20 years, born with cleft lip and palate).

Individuals could be disappointed with past treatment outcomes, sometimes because they did not know what to expect following a procedure and was facilitated parents and medical professionals.

I was always disappointed with the outcome [laughs] because I'd think I was going to be this, you know, perfect nose or perfect lip or perfect teeth and in reality, if I'd had the opportunity to discuss it, I'm sure they would have told me, "Well actually, no, we're giving you the best outcome not necessarily how you may view it or want it". So, I really feel that it was discussed between my parents and the doctors, and my parents went along with everything that the doctors and professionals suggested... (Female, 53 years, born with cleft lip and palate).

Even though some individuals didn't feel part of the decision-making process, there was evidence that, retrospectively, they understood the need for and benefits of treatment. It was also common for older individuals to have disengaged from cleft treatment services in adolescence but to accept the need for palate revision due to facial growth in adulthood. However, ongoing treatment that was deemed unnecessary or functionally disruptive was often unwelcome at a time when young adults are meeting new people and developing relationships in social and workplace environments.

I moved to London, met a whole new bunch of people, quite a lot of kind of weird and wonderful people, kind of it was just quite an exciting five years. And I think the kind of rollercoaster of that time combined with those, you know, being 18, 19, 20 etc., perhaps overshadowed any issues around having a cleft, to be honest. I was just doing stuff that came along in quick succession. Yeah and I had to have an operation when I was 20 to fill my palate, had the whole of my palate reworked. There were kind of options to do jaw alignment after that, and I kind of didn't because that surgery at 20 was well it wasn't a very pleasant experience for me. (Male, 36 years, born with cleft lip and palate).

\section{Expectations of treatment and communication}

As well as feeling uninvolved in the decision-making process, some individuals were dissatisfied with their experience of cleft treatment services for a variety of reasons including the nature of the patient-clinician relationship and differing values with regard to aesthetic outcomes.

The only thing I would say about my surgeon, especially in the latter stages of my treatment, was I felt like I wasn't really a person, I was more like a canvas, which I didn't particularly enjoy. You know, I'm thinking, "I'm a person, I've got a heartbeat, treat me like an individual - treat me like a human being rather than just a painting which needs, you know, improved on". That was the one thing that I really didn't like. And after my last operation my surgeon said, "Right, we can do a little more just to tidy the nose up", and I said, "I don't want any more. I don't want any more surgery", you know. (Female, 25 years, born with cleft lip and palate).

Some individuals reported that the emotional trauma prior or following cleft treatment was not recognised with regard to the psychological health of the individual.

And certainly, you know, every physical issue that somebody has, has a psychological, a mental and emotional consequence as well. You know, as a child with a cleft palate and lip there was no point at which anybody 
ever discussed with me or talked with me in any way that could be described as psychological or, you know, for my psychological health wellbeing or emotion wellbeing: that was never addressed with me. And anything I ever felt about how I looked or, you know, what was happening was very much, "Oh this is not right: we must fix it". And I personally don't like that approach. You know, it's different; it's not wrong. You know, people are just different. (Female, 41 years, born with cleft lip and palate).

Fortunately, some individuals had more satisfactory experiences of cleft treatment services as young adults. They felt that they had been given the opportunity to make an informed choice and in their own time.

I had known about it for quite a long time. But because it's an operation where you need to wait until you've stopped growing I can it was quite a long wait from finding out about it to then actually having it. So obviously, your cleft team explain it all to you and it's obviously your choice, it's not a necessary operation but like obviously beneficial. So, it was just brace work for quite a long time and then kind of meeting with the team and it was any questions I had they were more than happy to answer them, and I felt like really comfortable to go to them with cleft issues that I had, even if it was something really small and might be insignificant, but it's not like they were more than happy to answer it. So, the support network before it was preparation for it was really good. (Female, 19 years, born with cleft lip and palate).

\section{Re-engagement with treatment}

Dissatisfaction with past treatment was often an impetus for returning to treatment as an adult for both aesthetic and functional concerns. However, re-engaging with specialist cleft services had implications for individuals' expectations of treatment and outcomes.

One individual who had returned to treatment as adults believed that specialist professionals were disrespectful considering that they were discussing surgery that would ultimately change their facial appearance.

They were looking at my palate and I had this test, this machine, where I sat in this machine, and there was about eight of these people in this room, and students, and that's fine, but I wish they'd said to me, "We're just looking out to find out if your palate can't cope with this then", you know, I wish they'd explained more of what was going on and what could happen if this isn't right, or what could happen if that is wrong. You know, I just wanted to know more about the whole thing, not I mean nobody was wrong, but it could have been better. I just felt like you're talking about rearranging my face you know, it's not like, you know, reshaping a toe; it's my face [laughs]. (Female, 47 years, born with cleft lip and palate).

Some individuals also made a clear distinction be- tween having surgery for functional or aesthetic issues, and adjusting to facial change following cosmetic surgery conducted within the care pathway was sometimes a negative experience as a young adult.

I think there were essential ones to have, you know, if it was a palate problem or a functioning problem then it needed to be done. I appreciate that more now than I did [laughs] at the time. But there were times when were options for cosmetic things to happen, so the rhinoplasty, the implants and all of that kind of thing. And that actually had quite a negative effect on me afterwards. So, I mean there was one, I was 19 and I'd had rhinoplasty, implants and the scar taken all in one big operation. I'd been warned that I was going to look a bit different afterwards. I just, I couldn't cope afterwards. I saw my face and I just thought, "This isn't what I expected", not that I really knew what to expect; it was just, you know, kind of it's an adjustment to your face that you now have to reintroduce yourself to every day (Female, 25 years, born with cleft lip and palate).

Finally, a few individuals were fortunate to be able to access psychological services pre-and post-surgery in early adulthood and it was a much-valued source of support which was lacking in earlier years.

There's a really good psychology department at the hospital I went to. And after that they were really supportive in you know, helping me come to terms with, you know, my new face and whatever. I've really benefitted from the psychology department. I think if I'd had them earlier I would have done even better. And I think for my parents' sake as well I think we all would have benefited from it, you know, when I was younger, having that support. They were just really resourceful in helping you manage your emotions and how you're feeling about, you know, the surgery you've had or... and even getting you to think about the future, if you want more surgery, thinking more rationally about, "Is that really what I want or am I being influenced by this or that?" (Female, 20 years, born with cleft lip and palate).

\section{Health professionals' awareness of cleft}

Regular dental check-ups and oral hygiene practices are important for individuals born with CLP, thus a high level of awareness among individuals and health professionals is required.

Just keep an eye on it. And if there are surgeries, keep on top of your oral hygiene. If you're like me and my oral hygiene is fine, but there are a couple of times when my oral hygienist has just turned around and gone, "Right, you need to do this". If there is an issue they'll tell you, and tell you how to fix it. No just kind of, just kind of every three to six months I'll see them (Male, 19 years, born with cleft lip and palate).

However, individuals reported a lack of awareness 
and empathy from their GDPs and GMPs with regard to issues such as breathing difficulties and phobia that may be exacerbated in individuals born with CLP.

It was making me phobic of going to the dentist because I couldn't breathe and I also felt that the dentist I had thought I was being a bit of a wuss and didn't understand my condition ...... I think it was about two and a half years ago I was having some difficulties with going to the dentist because I couldn't breathe properly, and so when they put the water in your mouth I always felt like I was drowning because I couldn't breathe through my nose because what I found out was something had kind of collapsed on that side and it was preventing me from breathing (Female, 53 years, born with cleft lip and palate).

With the dentist, the first dentist I had here she talked to me like I didn't have a clue what I was talking about. And for someone who has lived with this for 41 years now I may not I certainly don't have a medical degree, but I'm fairly up on some of the issues, and I've talked to dentists an awful lot in my life. So, I fully expect that the dentist is going to have more information and more knowledge, but when they don't have any, and then tell me that I don't know what I'm talking about ... just I have no patience for that (Male, 41 years, born with cleft lip and palate).

Finally, individuals were vociferous in their views with regard to the role of GMPs and GPs and their understanding of CLP and specialist cleft services in the UK. As individuals drift away from specialist cleft services in early adulthood, registration with a dentist who has an awareness of CLP and the implications for re-engaging with specialist services is very important.

When I was 18 I was old enough to make the decision myself and I approached my dentist and she said, "Oh yes you can". [seek further treatment] and that's when I had the sort of lame orthodontics. I think I might have been in denial myself because, you know, you're facing your own face, sort of thing; it's quite hard. And I thought I had to pay: that's why I hadn't looked into it before. Because orthodontics over the age of 16 or whatever you had to pay, didn't you? So, I spoke to my dentist (in Forties) and she said, "Well no", she said, "you were born with a cleft, weren't you?" She recognised that. And, yeah, it was good, and she said, "Well if you were born with a cleft your healthcare is still with the NHS". And I was absolutely shocked (Female, 47 years, born with cleft lip and palate).

Other individuals, however, experienced great difficulty in getting GMPs and GDPs to listen to their concerns and refer for appropriate treatment in the health service.

The frustrations here are born out of a couple of times now dealing with either GPs or dentists who have no idea what it means to deal with someone with a cleft palate. And I find it incredibly frustrating to be fighting what they think is correct when I've been told, advised for years and years from specialists, on what is or what should be done and really having to stand up. And the initial reaction with any dental problem here, especially when I was on the NHS, was, "Well we'll just pull the teeth and we'll look and see and possibly put in some replacements later". And to get them to recognise what that means for someone who has always been I was always self-conscious about my looks, still am, and to be told, "We're going to take teeth out from the front of your mouth", is shocking and horrifying (Male, 41 years, born with cleft lip and palate).

\section{Discussion}

The aim of this qualitative study was two-fold; to explore: 1) Experiences of specialist cleft treatment during childhood and adolescence for individuals born with CLP and 2) Re-Engagement with specialist cleft services as an adult in the U.K. These aims were explored within the context of living with CLP and non-specialist health professional awareness of CLP. The thematic analysis led to the identification of 4 key themes: Living with a cleft as an adult (including professional support pre- and post-treatment), re-engagement with treatment as an adult (for aesthetic and functional reasons), Expectations of treatment and communication, Health Professionals' awareness of cleft (GMPs, GDPs and referral to specialist services).

Individuals born with CLP had concerns with regard to satisfaction with and adjustment to appearance after previous surgery, or other issues that were not adequately addressed in the 'cleft treatment pathway' during childhood. It was also clear from the interviews that many individuals born with CLP did not feel part of the decision-making process as child patients. This finding may be partly due to older individuals experiencing a more 'paternalistic' healthcare system that was prevalent when they were teen-aged or young adults. Unfortunately, younger individuals had also not felt that their views and feeling were sufficiently considered in their consultations with health professionals. This echoes a recent qualitative study with 52 adults born with CLP found that some participants questioned the value and necessity of surgical revision and treatment decision-making was influenced by other peoples' opinions and consequently led to the exacerbation of confusion and/or distress. This may be a particular burden at a time when adolescents face challenges in the development of self-confidence. Hence, this overt emphasis on surgery in adolescence may be driving a tendency for young adults to drift away from cleft services in early adulthood and to return later for treatment of their own volition and autonomy.

Consistent with previous research some of the individuals interviewed had unmet expectations relating to functional concerns such as breathing or nasal symp- 
toms [13], speech, and audiology [14,15]. Furthermore, patients required restorative dental treatment and/or referral to orthodontist services [17]. As adults, some individuals had mixed experiences with regard to the extent that non-specialist professionals such as GDPs and GPs were able to recognise and support individuals presenting with issues that were related to being born with CLP. Thus, the present findings have implications for health professionals with regard to communicating with adults with cleft and support the notion that adults born with CLP may want or need further multidisciplinary treatment for symptoms associated with their cleft, and it is important for GPs and GDPs [16] to understand these needs and to refer to the specialist services that do exist.

It has been suggested that re-engaging with treatment as an adult can evoke a strong emotional reaction and the need for subsequent psychological intervention [8]. However, there was also little data relating to the availability of psychological support for individuals considering re-engagement with specialist cleft treatment services. Indeed, individuals were not accessing psychological services due to a lack of awareness of availability for adults as it was considered for families and younger patients. Some individuals had sought private counselling for dealing with emotional tension that individuals had attributed to cleft related issues or had found support through online organisations such as Adult Voices network. Individuals recalled experiences of treatment that were often felt to challenge their self-perception suggesting that treatment could be driven by agendas favouring surgical intervention. Indeed, appearance altering surgery may invoke considerable emotional and psychological adjustment but individuals come to accept their appearance such that decisions for further intervention may be based on a delicate cost-benefit balance.

\section{A strengths and limitations}

This study reports the personal and individual experiences of a sample of individuals born with CLP across the U.K. However, it should be noted that older individuals born with CLP would not have been treated within the centralised cleft service introduced in 1998 and so there is a chance that we have heard about temporal discrepancies in treatment practices $[6,20]$. Therefore, there is a need for future qualitative research to focus on individuals who have or recent experience of the 'cleft treatment pathway' in order to better evaluate post-CSAG experiences of treatment. However, as noted above the lack of involvement in the decision-making process was reported by individuals across the age spectrum including some aged less than 25 years of age: this experience, at least, appears not to have changed over time. However, it should be considered that interventions in cleft care are improving, but societal influences and celebrity culture that emphasise 'perfect ap- pearances' may make it even harder for those that fall outside of the norm.

There is potential 'self-selection' in that a small number of individuals participated because they had particular experiences to disclose. However, an experienced qualitative researcher (AS) was sensitive with regard to engagement with the interviewees and through asking open questions and careful probing served to minimise the opportunity to elicit particularly negative experiences. Furthermore, individuals were encouraged to tell their 'story' rather than imposing pre-conceived hypotheses on the data, and serves to contextualise the findings in the narrative of daily life. However, as the data represents the experiences of a wide range of individuals and some who had not been part of the 'cleft treatment pathway' within the NHS it is not certain that data saturation was achieved and limits the generalisability of the present findings.

Finally, strength of this study was that most of the interviews were conducted in the context of face to face video interviews, which is unique to CLP research. However, some individuals elected for telephone interviews for geographical, logistical or preference reasons. It has been suggested that interviews conducted by telephone can hinder the establishment and maintenance of rapport between an interviewer and interviewee and prevent the interviewer from attending to non-verbal cues [21]. However, it is worth noting that in the present study the shortest interview was conducted face to face (22 minutes) in contrast to the longest telephone interview (48 minutes) suggesting that mode of interview did not adversely impact on maintenance of interview.

\section{Implications for GMPs and General Dental Practi- tioners (GDP) working in primary care}

Individuals born with CLP who require a re-referral to the cleft specialist services will often present to professionals in primary care e.g. their GMP or GDP. However, GMPs and GDPs may lack knowledge and the specific needs of an individual born with CLP. Undergraduate training in CLP for GDPs and more so for GMPs is fairly limited in the UK. Similarly, post graduate specialist trainees in relevant dental specialities such as restorative and paediatric dentistry and orthodontics is also limited, and is seen more as a post specialist training extended competency within the training pathways in the U.K. This extended training will only be present in certain specialist units and will depend on the individual interests of the specialist trainers and trainees. Yet adult CLP patients may have restorative and orthodontic needs which require specialist input. To access these specialist services will require referral from the GMP/GDP to whom the patient will initially present. This in turn will require those in primary care to have sufficient knowledge of the ongoing treatment needs of these patients, as well as an awareness of centralised cleft services and the referral process. Only an informed 
GMP/GDP will be in a position to facilitate and refer an individual born with CLP to relevant services.

Finally, there is a lack of psychological support for adults born with CLP who may be disatisfied with their appearance, speech or experience impaired functionality. Despite disparity in commissioning of cleft services [22] the present data suggests there is a need for continued access to psychological services both pre-and post-intervention as this is one service that is currently overlooked. Thus, in addition to signposting and referrals from non-specialists, individuals born with CLP may also require psychological support when re-entering cleft treatment services [8].

To conclude this qualitative study, it was clear that individuals born with CLP have concerns with regard to satisfaction and psychological adjustment to treatment that were not adequately addressed by specialist professionals.

Furthermore, following specialist cleft care these individuals experienced a low level of awareness of the issues associated with CLP from non-specialists in the health service such as GDPs and GMPs. In particular, the adverse psychological impact for individuals undergoing treatment for CLP would appear to be associated with poor communication prior to surgery such that there is a need to manage expectations of treatment in the context of the life stage of the individual concerned. Thus, future research would be well placed if it focused on the perceived psychological needs and support received by individuals considering returning to or referred to cleft services in adulthood.

In order to improve non-specialist care and access to specialist care for individuals born with CLP there is a need for further training of GMPs and GDPs. This training could be provided at undergraduate level and followed by further Continuing Professional Development (CPD) opportunities to raise awareness of the issues faced by individuals born with CLP.

\section{References}

1. Stanier P, Moore GE (2004) Genetics of cleft lip and palate: syndromic genes contribute to the incidence of non-syndromic clefts. Hum Mol Genet 13: 73-81.

2. Mossey P, Castilla E (1992) Global registry and database on craniofacial anomalies. Report of a WHO Registry Meeting on Craniofacial Anomalies. Geneva, Human Genetics Programme, Management of Non-Communicable Diseases, World Health Organization, 16-20.

3. WHO (2003) International Centre for Birth Defects (ICBD) of the International Clearinghouse for Birth Defects Monitoring Systems in collaboration with European Surveillance of Congenital Anomalies (EUROCAT) in cooperation with Human Genetics Programme World Health Organization.

4. Fitzsimons K, Deacon S, Copley L, van der Meulen J (2013) CRANE Database.

5. Fitzsimons KJ, Mukarram S, Copley LP, Deacon SA, van der Meulen JH (2012) Centralisation of services for children with cleft lip or palate in England: a study of hospital episode statistics. BMC Health Serv Res 12: 148.
6. CSAG (1998) Cleft lip and/or palate. Report of CSAG committee. London, HMSO.

7. Frey B, Argent A (2004) Safe paediatric intensive care. Part 2: workplace organisation, critical incident monitoring and guidelines. Intensive Care Med 30: 1292-1297.

8. Stock NM, Feragen KB, Rumsey N (2015) "It Doesn't All Just Stop at 18": Psychological Adjustment and Support Needs of Adults Born with Cleft Lip and/or Palate. Cleft Palate Craniofac J 52: 543-554.

9. Richman LC (1983) Self-reported social, speech, and facial concerns andpersonality adjustment of adolescents with cleft lip and palate. Cleft Palate J 20: 108-112.

10. Turner SR, Rumsey N, Sandy JR (1998) Psychological aspects of cleft lip and palate. Eur J Orthod 20: 407-415.

11. Strauss RP, Broder H, Helms RW (1988) Perceptions of appearance and speech by adolescent patients with cleft lip and palate and by their parents. Cleft Palate J 25: 335-342.

12. Searle A, Neville P, Waylen A (2017) Psychological growth and well-being in individuals born with cleft: An application of self-determination theory. Psychol Health 32: 459-482.

13. Morén S, Mani M, Lundberg K, Holmström M (2013) Nasal symptoms and clinical findings in adult patients treated for unilateral cleft lip and palate. J Plast Surg Hand Surg 47: 383-389.

14. Flynn T, Lohmander A (2014) A longitudinal study of hearing and middle ear status in individuals with UCLP. Otol Neurotol 35: 989-996.

15. Flynn T, Möller C, Lohmander A, Magnusson L (2012) Hearing and otitis media with effusion in young adults with cleft lip and palate. Acta Otolaryngol 132: 959-966.

16. Hamlet C, Harcourt D (2015) Older adults' experiences of living with cleft lip and palate: a qualitative study exploring aging and appearance. Cleft Palate Craniofac J 52: e32-e40.

17. Chuo CB, Searle Y, Jeremy A, Richard BM, Sharp I, et al. (2008) The Continuing Multidisciplinary Needs of Adult Patients with Cleft Lip and/or Palate. Cleft Palate Craniofac J 45: 633-638.

18. Sharif MO, Callery P, Tierney S (2013) The perspectives of children and young people living with cleft lip and palate: a review of qualitative literature. Cleft Palate Craniofac J 50: 297-304.

19. Braun V, Clarke V (2006) Using thematic analysis in psychology. Qualitative Research in Psychology, 77-101.

20. Scott JK, Leary SD, Ness AR, Sandy JR, Persson M, et al. (2014) Centralization of Services for Children Born with Orofacial Clefts in the United Kingdom: A Cross-Sectional Survey. Cleft Palate Craniofac J 51: e102-e109.

21. Novick $G(2008)$ Is there a bias against telephone interviews in qualitative research? Res Nurs Health 31: 391-398.

22. Searle A, Scott JK, Sandy J, Ness A, Waylen A (2015) Clinical directors' views of centralisation and commissioning of cleft services in the U.K. BMC Oral Health 15: 12. 
Appendix 1: Topic Guide for Interviews with Individuals Born with Cleft Lip and/or Palate.

\section{Background}

How would you describe yourself to someone who hadn't met you?

Who do you live with?

What are your interests - what do you do in your spare time?

\section{School experience}

Can you tell me about your school years?

What sort of challenges did you face at school?

PROMPT:

- Emotional

- Educational attainment

- Identity and relationships with other pupils and teachers

- Satisfaction with appearance

- Awareness of others

- Social and cultural differences

- Parental guidance/family life

\section{Everyday life}

Can you tell me about your life outside of school?

- Emotional

- Identity and relationships with others

- Satisfaction with appearance

- Awareness of others

- Social and cultural norms/differences

- Parental guidance

\section{Cleft treatment}

What can you remember about your past treatment for cleft - what are your earliest memories?

To what extent have you felt/do you now feel satisfied with treatment?

What impact did it have on your life in school?

What particular treatment specialisms have had the biggest impact?

- On your schooling?

- On your social experience?

- On your employment prospects and (if appropriate) professional role?
PROMPT:

- Surgery/cosmetic correction

- Orthodontics

- Speech therapy/audiology

- Psychological services

- Other

\section{Social experience - post school}

Can you tell me about your social experience since leaving school?

- Emotional aspects

- Identity and relationship with others

- Romantic relationships

- Satisfaction with appearance

- Awareness of others treating you differently

- Social and cultural differences

- Parental guidance/family life

\section{Post school experience and employment}

What have you done since leaving school?

Are you currently employed?

If so, can you tell me about your experiences of finding employment?

- If still in education, can you tell me about your future employment plans?

- Can you tell me about your opportunities for employment and progression within the workplace?

- What plans do you have for the future?

\section{Strategies for overcoming cleft related difficulties}

In what ways have you been able to overcome any social difficulties relating to having cleft?

Are there any issues relating to being born with a cleft that could have been addressed differently?

What advice do you have for other young people who have a cleft lip and/or palate?

To what extent is there a possibility that a future child of yours may be born with a cleft lip and/or palate?

- How do feel about this?

- How would you summarise your experience of living with a cleft lip and/or palate?

- Is there anything we haven't covered that you want to talk about? 